\title{
Le JATBA et ses ancêtres
}

Essai d'histoire de la revue d'un laboratoire du Muséum national d'histoire naturelle

JATBA and its ancestors: Essay on the history of a Muséum National d'Histoire Naturelle laboratory's journal

\section{Catherine Hoare}

\section{OpenEdition}

\section{Journals}

Édition électronique

URL : http://journals.openedition.org/ethnoecologie/673

DOI : 10.4000/ethnoecologie.673

ISSN : 2267-2419

\section{Éditeur}

Laboratoire Eco-anthropologie et Ethnobiologie

\section{Référence électronique}

Catherine Hoare, "Le JATBA et ses ancêtres », Revue d'ethnoécologie [En ligne], 1 | 2012, mis en ligne le 30 juin 2012, consulté le 30 avril 2019. URL : http://journals.openedition.org/ethnoecologie/673 ; DOI : 10.4000/ethnoecologie.673

Ce document a été généré automatiquement le 30 avril 2019.

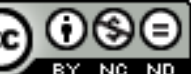

Revue d'ethnoécologie est mis à disposition selon les termes de la licence Creative Commons Attribution - Pas d'Utilisation Commerciale - Pas de Modification 4.0 International. 


\title{
Le JATBA et ses ancêtres
}

\section{Essai d'histoire de la revue d'un laboratoire du Muséum national d'histoire naturelle}

\author{
JATBA and its ancestors: Essay on the history of a Muséum National d'Histoire \\ Naturelle laboratory's journal
}

\section{Catherine Hoare}

\section{Introduction}

1 En 1953, Auguste Chevalier (1873-1956), dans le bulletin numéro 373-374 de novembredécembre de la revue du laboratoire d'Agronomie coloniale, écrit: «La Revue Internationale de Botanique Appliquée et d'Agriculture tropicale (R.B.A.) cesse de paraître avec ce numéro ».

2 Son fondateur vient d'avoir quatre-vingts ans et n'est plus en mesure d'en assurer la publication; il décide qu'après trente-trois années de parution régulière, au cours desquelles il en a assuré la gestion, la rédaction, et a été l'auteur d'une grande partie du contenu, elle doit disparaître.

"Cher abonné, cher lecteur...» C'est ainsi que Jacques Barrau (1925-1997) débute le volume XXXIV du Journal d'Agriculture traditionnelle et de Botanique appliquée (JATBA) de 1987, avant-dernier d'une série, pour annoncer un énième tournant dans la trajectoire de la revue du laboratoire d'Ethnobiologie-Biogéographie. Le JATBA doit se restructurer et une nouvelle politique éditoriale est proposée. Plus qu'un tournant c'est un sommeil de six années qui commence : le cri du phénix qui va périr et puis, peut-être... renaître par sa seule volonté, force interne. Comparer le JATBA à un phénix, oiseau qui renaît de ses cendres, on y pense tout de suite en se penchant sur son histoire, mais on ignore parfois à quel point la comparaison est pertinente ${ }^{1}$. Le phénix est un oiseau mais c'est aussi un genre de palmier. Un animal, un végétal, un mythe, tous les ingrédients de l'ethnozoologie et de l'ethnobotanique sont ici présents. 
4 De 1921 à 2008 la revue a eu divers titres, a revêtu divers aspects; son contenu et sa gestion ont bien sûr évolué en fonction des chercheurs qui s'en occupaient au sein du laboratoire qui l'a vu naître.

C'est cette histoire que nous allons raconter et tenter d'analyser ici en considérant d'une part la Revue de Botanique Appliquée, sa création et sa gestion par Auguste Chevalier et d'autre part l'après Chevalier, la naissance et l'évolution du Journal d'Agriculture Tropicale et de Botanique Appliquée jusqu'au JATBA Revue d'Ethnobiologie nouveau cru.

\section{La gestation - Le contexte éditorial et scientifique}

6 L'idée d'Auguste Chevalier de créer la Revue de Botanique appliquée est à rechercher dans les contextes scientifique, politique et éditorial de la fin du XIX ${ }^{e}$ et du début du xx $x^{e}$ siècles largement tournés vers les explorations des colonies en Afrique notamment et l'installation et la structuration d'une agriculture dans les colonies.

7 Depuis 1897 paraît la Revue des Cultures coloniales publiée par Milhe-Poutingon (1856-1920), directeur du service de l'Afrique à l'Union coloniale française ${ }^{2}$. La même année Auguste Chevalier a vingt-quatre ans et entre comme boursier de doctorat au Muséum d'Histoire Naturelle de Paris pour terminer une thèse en botanique sur les Myricacées, commencée à l'Université de Lille.

8 L'année suivante Chevalier est sollicité pour assurer la prospection botanique de la mission scientifique et militaire du Général de Trentinian qui va durer dix-sept mois ${ }^{3}$.

En 1901 le Jardin colonial de Nogent-sur-Marne sous l'égide du Ministère des Colonies édite l'Agriculture pratique des Pays chauds, et Jean Vilbouchevitch ${ }^{4}$ fonde le Journal d'Agriculture Tropicale (Jd'AT).

De 1902 à 1904, Auguste Chevalier prospecte en Afrique centrale, c'est la Mission Oubangui-Chari-Lac Tchad. À cette époque, le botaniste est déjà l'un des principaux collaborateurs du Journal d'Agriculture Tropicale. Ses missions d'exploration et de collecte s'enchaînent de 1905 à 1913 avec le but d'identifier les plantes utiles, exploitables dans les colonies. Il effectue quatre autres voyages successifs à travers l'Afrique tropicale d'où il ramène environ 17000 numéros d'herbier 5 . À cette période il publie abondamment dans diverses revues comme par exemple les Comptes rendus de l'Académie des Sciences, La Géographie, le Bulletin du Muséum d'Histoire Naturelle, le Bulletin de la Société des Études coloniales et maritimes.

11 En 1911, à la suite d'un accord entre le ministère de l'Instruction publique et le ministère des Colonies, un Laboratoire d'Agronomie coloniale de l'École des Hautes Études est créé et installé au Muséum. Auguste Chevalier en est nommé directeur et c'est là qu'il va « faire son nid »: déposer ses collections, rédiger ses récits d'exploration et exploiter les résultats de ses prospections botaniques.

En 1912 le Ministère des Colonies crée la Mission permanente d'Étude des Cultures et des Jardins d'essais coloniaux et Chevalier qui a déjà fait plus de dix ans de terrain en Afrique en est nommé directeur; il est obligé de "porter ses regards sur les autres colonies françaises » (Urbain 1947). Il partira alors pour l'Indochine, Java, Ceylan.

Il a déjà publié environ soixante-dix articles et notes dans le Journal d'Agriculture Tropicale dont il devient directeur en 1913. Pour marquer sa prise en main de cette revue il publie, en supplément au numéro 146, un Bulletin du Laboratoire d'Agronomie coloniale. 
14 1914, c'est la guerre, les missions sont interrompues. Auguste Chevalier sert aux Hôpitaux sénégalais de Menton et il participe à la création en 1915 du Comité d'assistance aux soldats coloniaux. Le Journal d'Agriculture Tropicale publie sept fascicules en 1914 puis s'interrompt comme beaucoup de publications.

15 En 1916, « [le Gouverneur général de l'Indochine, Albert] Sarraut appelle [...] Chevalier en Indochine pour proposer une nouvelle organisation des services agricoles. [Il en revient] avec de vastes ambitions pour l'organisation des recherches agricoles dans l'Empire. [...] $\mathrm{Au}$ lendemain de la guerre, Sarraut fonde l'Institut scientifique de Saigon. Mais Chevalier abandonne la direction de cet ambitieux organisme de recherche de biologie et d'agronomie pour asseoir sa situation en métropole » (Bonneuil 1996).

16 Les éditions reprennent pour la plupart des titres de périodiques dès 1918. En avril le Journal d'Agriculture Tropicale publie le bulletin 158 et le numéro 159 ne sera publié qu'en mai 1919 annonçant un programme éditorial précis et faisant appel aux abonnés. C'est pourtant la fin de ce titre... Le Phénix périt une première fois.

17 Depuis fin 1917, Auguste Chevalier s'est installé dans son laboratoire au Muséum, dans le bâtiment de Cuvier. Dans un article de 1921, Chevalier (1921 : 3-11) expose les activités de son laboratoire et précise la politique qui sous-tendra son action scientifique tout au long de sa carrière, à savoir: réunir une documentation imprimée constamment à jour (ouvrages, articles, rapports en français ou autres langues, revues); enrichir les collections naturalistes (herbiers, objets, graines, produits transformés) ; et enfin rendre compte des résultats de recherches sur ces collections ou de lectures en publiant abondamment. Les trois supports qui constituent l'ossature de son œuvre et de son laboratoire se mettent en place : la bibliothèque, les collections et la revue qu'il va créer.

\section{La naissance - Le programme - L'accident}

18 C'est ainsi que le 15 mai 1921 paraît le volume I, numéro 1 de la Revue de Botanique appliquée et d'Agriculture coloniale sous-titré : Bulletin du Laboratoire d'Agronomie coloniale ${ }^{6}$. Dans un article intitulé "Au lecteur », Auguste Chevalier précise la forme et le contenu qu'elle aura en la démarquant nettement du Journal d'Agriculture Tropicale, dont la publication est « pour le moment suspendue » dit-il : elle ne traitera « pas exclusivement de l'agriculture tropicale, [...] mais aussi de l'horticulture coloniale, de la phytopathologie, des forêts et des bois coloniaux, ...toutes questions qui relèvent du domaine de la Botanique appliquée » (Chevalier 1921 : 1-2). La revue est présentée comme une suite au Bulletin du Laboratoire d'Agronomie coloniale auquel nous avons fait référence plus haut, paru en 1913. Chevalier insiste sur l'originalité de sa publication par rapport à celles de l'époque et notamment l'Agriculture pratique des Pays chauds qui est devenue en 1913 l'Agronomie coloniale publiée par le Jardin colonial de Nogent-sur-Marne. Le conflit de l'époque entre les différentes institutions responsables de la formation des agronomes coloniaux, notamment entre le Muséum et Nogent, est matérialisé par une rivalité ou tout au moins un désir de se démarquer de leurs revues respectives ${ }^{7}$.

19 Trois bulletins sont publiés en 1921 et 1922 commence avec le numéro 5 bizarrement ${ }^{8}$. Dès la deuxième année le sous-titre a changé : Organe de l'Association internationale de Botanique appliquée et du Laboratoire d'Agronomie coloniale ${ }^{9}$. Le Bulletin du Laboratoire n'aura eu qu'une courte renaissance! En réalité Chevalier semble considérer désormais que la revue elle-même en tient lieu. Dans "Notre programme », il trace une ligne 
éditoriale; il inscrit la revue dans le contexte international des revues consacrées aux mêmes thématiques: "C'est à la France et aux colonies françaises que s'adresse en premier lieu la Revue de Botanique appliquée et d'Agronomie coloniale » est-il écrit en gras dès les premières pages.

«Le but que nous nous proposons sera de tenir le public au courant des travaux scientifiques concernant les plantes utiles, l'agriculture, les produits forestiers en publiant des travaux originaux, des dossiers, des analyses bibliographiques... La $\mathrm{RBA}^{10}$ se propose de renseigner ses lecteurs sur les progrès de l'agriculture et de l'exploitation forestière dans la métropole et dans les colonies, sur les possibilités économiques et le commerce auxquels les productions tropicales donnent lieu ». Douze fascicules sont publiés en 1922 pour 850 pages environ.

Dès 1923 Chevalier dresse un premier bilan de la publication: il souligne les étapes franchies, l'augmentation progressive du nombre de pages de chaque bulletin et aussi les échanges avec les revues étrangères. Le sous-titre a encore changé : Organe mensuel de l'Agriculture scientifique pour la France \& ses Colonies publié par le Laboratoire d'Agronomie coloniale de l'École des Hautes Études. En 1924 un programme annuel est annoncé : « ... nous nous efforcerons de conserver à la Revue de Botanique appliquée sa tenue scientifique tout en la maintenant constamment dans le domaine des applications agricoles pour la France et pour son domaine colonial» (Chevalier 1924). La bibliographie qui occupe un dossier déjà important, est structurée désormais en trois parties : Bibliographie sélectionnée (recensions détaillées); Travaux récents sur l'Agriculture en général et sur les plantes utiles des pays tempérés et enfin Travaux récents sur les cultures et les plantes des pays chauds. Comme dans le programme et dans le sous-titre, on insiste sur les deux grands pôles biogéographiques visés : la France et les pays tempérés d'une part, les pays tropicaux d'autre part.

1925 est un tournant à plus d'un titre pour la RBA. Tout d'abord un supplément lui est annexé : les Actes et comptes rendus de l'Association Colonies-Sciences (six bulletins). Cette association a été fondée en janvier pour « unir, grouper, pour un effort commun, les techniciens de l'agronomie coloniale, les laboratoires, Instituts coloniaux et établissements similaires ». (NDLR 1925). Auguste Chevalier en est le secrétaire général. "Elle rassemble des scientifiques et des techniciens de diverses disciplines, ainsi que diverses personnalités des lobbies économiques et politiques coloniaux. » (Bonneuil 1996).

la revue se structure différemment. Des «instructions aux auteurs " très détaillées sont publiées sur 14 pages! (Chevalier 1925). Le contenu des bulletins s'organise en quatre parties: Études et dossiers présente de courtes monographies paraissant parfois sur plusieurs bulletins; Notes et Actualités réunit des articles sur des sujets de recherche en cours; la Bibliographie rend compte des publications françaises et étrangères avec recension bien souvent et enfin, dans Nouvelles et Correspondances, sont publiées des notices nécrologiques ou des nouvelles de scientifiques en mission ou de correspondants. Le sous-titre de la revue disparaît.

Mais l'événement le plus important qui intervient cette année-là est le redoutable incendie qui ravage le laboratoire le 6 juin 1925. Les conséquences seront déterminantes pour la suite de la Revue : deuxième manifestation probante du Phénix. 


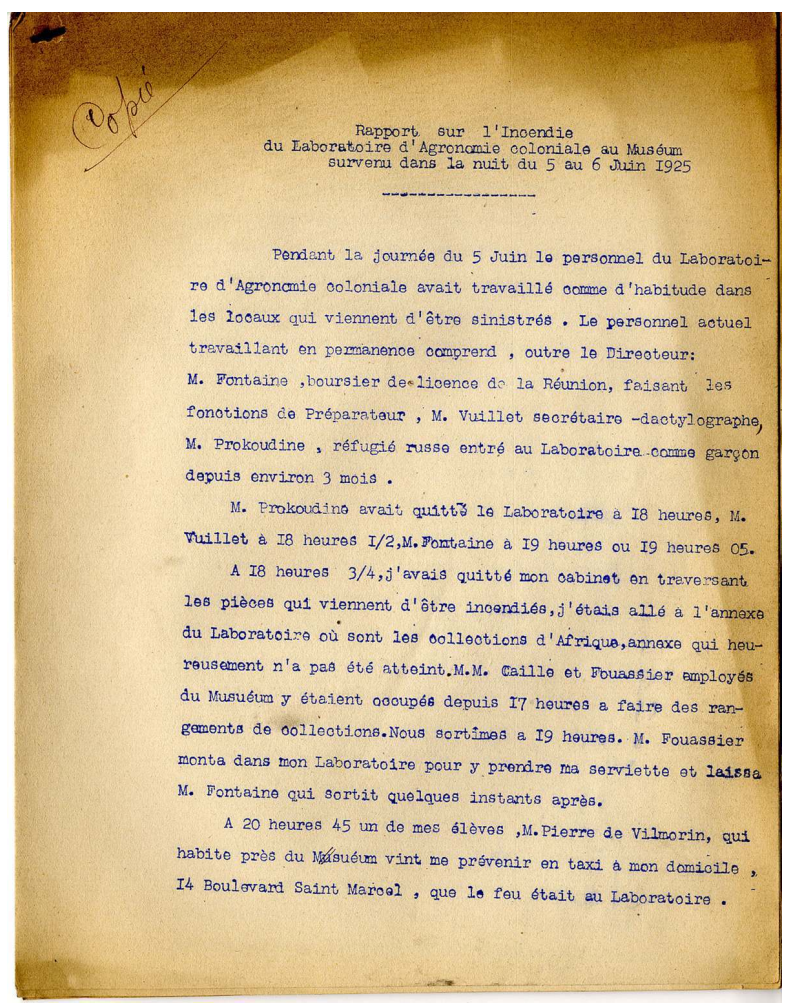

Archives laboratoire d'Ethnobiologie, Muséum national d'histoire naturelle, Paris

En 1926, le numéro 2 (numéro spécial) du Bulletin du Laboratoire d'Agronomie coloniale consacre vingt-huit pages à l'incendie et à ses conséquences. Un Comité de patronage du Laboratoire est créé, présidé par la veuve de Philippe de Vilmorin, dirigeant des établissements Vilmorin et ami de Chevalier, née Mélanie de Dortan. Il se charge de réunir des fonds pour reconstruire le laboratoire, reconstituer la documentation et assurer désormais une grande partie des dépenses liées à la revue.

\section{L'Âge d'or}

Jusqu'à la deuxième guerre mondiale, la revue connaîtra son âge d'or, son contenu va croissant (voir courbes en annexe), plus de mille pages certaines années. Son financement est assuré par le Comité de patronage du laboratoire mais la lecture des procès-verbaux des séances du Comité montre que la bataille et l'engagement de Madame de Vilmorin et d'autres membres sont déterminants pour trouver de nouveaux fonds.

Chevalier assure une grande partie des articles, des éditoriaux, des notices nécrologiques et recensions bibliographiques (voir annexe)

28 En 1929, un changement important intervient dans le titre: Agriculture coloniale, devient Agriculture tropicale. Chevalier justifie ce changement :

" Aujourd'hui la revue est lue dans la plupart des pays chauds, et elle compte des amis dans toutes les contrées tropicales et subtropicales de l'Ancien et du Nouveau Monde. Elle a pris le caractère international que nous souhaitions lui donner et de ce fait le mot " colonial " qui figure dans son titre ne répond plus complètement à ses buts. 
Récemment au cours d'un voyage au Brésil, nous avons pu nous apercevoir en effet, que l'appellation "d'agriculture coloniale" convient mal quand on l'applique aux divers pays de l'Amérique du Sud qui ont été autrefois des "colonies”, mais qui n'en sont plus, à l'exception des Guyanes.

En Angleterre et aux États-Unis, le mot “colonial” n'est plus que rarement employé dans les publications de langue anglaise, et en France même, il a un sens imprécis, puisqu'il désigne aussi bien des pays de protectorat, des pays à mandat, et même l'Algérie qui n'est autre, par sa forme de gouvernement, qu'une partie de la France située au-delà de la Méditerranée.

Déjà l'an dernier le Conseil Scientifique International de l'Institut d'Agriculture de Rome rejeta l'appellation d'Agriculture coloniale appliquée à l'agriculture des pays chauds et adopta l'expression "Agriculture tropicale et subtropicale " pour lui être substitué. » (Chevalier 1929) de quatre pages environ paraissent en pagination continue avec les bulletins de la RBA, le premier avec le numéro 137 de 1933 et le dernier avec le numéro 149 de 1934.

\begin{abstract}
«Dans la séance du 15 novembre 1932 du Comité de patronage du Laboratoire d'Agronomie coloniale, M. Aug. Chevalier proposa de réunir périodiquement à son laboratoire les coloniaux de passage à Paris et les personnes s'intéressant à la flore des colonies, aux forêts et aux cultures tropicales et de publier dans la R.B.A. un compte-rendu sommaire de ces réunions. Ce serait un moyen de faire connaître à l'extérieur les travaux qui se poursuivent sous les auspices du Muséum d'Histoire naturelle pour l'étude et la mise en valeur de notre domaine colonial.

Monsieur Lemoine, directeur du Muséum et vice-président du Comité conseilla à M. Chevalier de s'entendre à ce sujet avec ses collègues MM. Humbert, professeur à la Chaire de Phanérogamie du Muséum, Allorge professeur à la Chaire de Cryptogamie et Guillaumin, professeur à la Chaire des Cultures. [...] [Ils] se sont mis d'accord pour donner à ce groupement le nom d'Association des Botanistes du Muséum . Les réunions auront lieu quatre fois par an, à 17 heures, au Laboratoire d'Agronomie coloniale, le deuxième mardi des mois de janvier, avril, juillet et octobre. [...]

Par les comptes-rendus sommaires nous tiendrons les lecteurs de la Revue de Botanique appliquée au courant des travaux de botanique pure et de chimie intéressant les colonies qui se poursuivent au Muséum et nous signalerons également les travaux de caractère scientifique qui s'effectueront dans le Domaine colonial français. À cet effet nous dépouillerons chaque mois le Bulletin du Muséum et les autres publications scientifiques pouvant intéresser les colonies. » (CR 1933).
\end{abstract}

31 Cette même année, Monsieur Paul Lemoine, directeur du Muséum, a été intégré dans le Comité de patronage du Laboratoire d'Agronomie coloniale ; au cours de la séance du 14 décembre il fait remarquer que «Le comité de patronage du laboratoire d'agronomie coloniale, d'après son titre, semble n'apporter son concours qu'au laboratoire relevant de l'école pratique des hautes études et non à la Chaire créée postérieurement au Muséum sous le nom de Chaire des productions coloniales d'origine végétale. Il sait qu'il n'en est rien, mais néanmoins il pourrait résulter dans l'avenir des inconvénients de cette dualité d'appellation. Monsieur Lemoine n'est pas d'avis que le Comité change de nom, il lui paraît préférable de faire changer par l'Administration, si cela est possible, l'appellation de la chaire du Muséum, en la faisant désigner sous le nom de Chaire d'Agronomie coloniale. De cette manière, il n'y aurait aucune ambiguïté. Le comité laisse toute 
initiative à messieurs Lemoine et Chevalier pour poursuivre les négociations. » (Séance du 14 décembre 1932, A. de Vogüe [1928-1941]).

Les négociations en question ont abouti puisque la Chaire associée au Laboratoire est devenue Chaire d'Agronomie coloniale officiellement en 1933 donc sans doute dans les tout derniers jours de l'année puisque le procès-verbal ci-dessus date du 14 décembre. Cependant le décalage subsiste avec le titre de la revue qui a évolué en "Agronomie tropicale » depuis 1929, ce que la chaire ne fera qu'en novembre $1958^{11}$.

En 1936 apparaît un secrétaire de rédaction en la personne de P. Tissot dont le nom et la fonction sont mentionnés en couverture. A. Chevalier a repris ses missions ${ }^{12}$ et il est nécessaire de prendre un rédacteur permanent pour la revue. P. Tissot a publié une cinquantaine d'articles dans la RBA entre 1931 et 1940. Il contribue également aux recensions bibliographiques. Il est rémunéré par le Comité de patronage du Laboratoire à partir de mars $1934^{13}$. Il gagne 1040 francs par mois. Dans sa séance du 3 juillet 1936, le Comité évoque sa situation :

«Sur la proposition de Monsieur le Professeur Chevalier, le Conseil examine la situation de son collaborateur M. Tissot.

Il est envisagé, en premier lieu, que le Comité verse à l'État un montant en capital suffisant pour que le revenu puisse en servir à appointer un préparateur qui, en l'espèce, serait $\mathrm{M}$. Tissot, dont les fonctions deviendraient ainsi permanentes.

Après échange de vues, le Conseil décide de verser directement à $\mathrm{M}$. Tissot une mensualité de 1.500 frs et de prendre à sa charge une cotisation aux Assurances Sociales, à titre de garantie, ainsi que d'effectuer des versements correspondants à la Caisse nationale des Retraites, de manière à assurer à $\mathrm{M}$. Tissot des avantages équivalents à ceux qu'il pourrait retirer de sa nomination officielle comme préparateur.»

De fait, à partir d'octobre 1936, le traitement de Tissot passe à 1535 francs par mois et ceci jusqu'en avril 1937.

\section{La Seconde Guerre mondiale et ses suites}

Progressivement à partir de 1940 le nombre de pages va diminuer pour atteindre seulement 260 pages en 1945 (on était à près de 1200 pages en 1935). La revue n'est plus mensuelle. En 1941 les Actes et Comptes rendus de l'Association Colonies-Sciences cessent de paraître. Il y a pénurie de papier et Chevalier en fait état régulièrement. En 1944 il écrit douze des trente et un articles publiés. À soixante et onze ans il porte encore entièrement sa revue sur ses épaules.

\section{Regrets}

Dès la fin de la guerre un nouveau bilan et aussi un programme sont consignés dans les premières pages du volume de 1945. Régulièrement A. Chevalier a fait de ses éditoriaux des programmes ou des bilans ${ }^{14}$. Mais cette année est particulière, il faut faire le bilan humain également. "Espérons qu'en Indochine aujourd'hui délivrée, nous pourrons retrouver vivants nos amis et recevoir des nouvelles de tous ceux de nos collaborateurs qui s'y trouvaient lorsque ce pays fut traitreusement envahi par le Japon. » Il énumère tous ses anciens correspondants morts pendant la guerre. Il cite: le Britannique Sir Arthur Hill, directeur de Kew, le Russe N.I. Vavilov, le Belge Leplae et l'Italien Scaetta pour ne parler que des plus connus. Puis il ajoute: «Puisse cette liste ne pas s'allonger 
encore quand nous serons mieux informés des événements survenus à l'étranger ou dans nos colonies. Il nous faut hélas resserrer les rangs. »

\section{Si tous les gars du monde... L'enthousiasme retrouvé}

En 1946, un nouveau départ est donné avec un titre modifié: Revue Internationale de Botanique appliquée et d'Agriculture traditionnelle. Auguste Chevalier annonce six fascicules de quatre-vingts pages par an. "Elle [la Revue] s'occupera de tous les problèmes concernant l'agriculture des pays chauds et de toutes les questions connexes à la botanique, relatives à l'agriculture, à l'horticulture, les forêts, les pâturages, la phytopharmacie exotique, l'écologie appliquée, la systématique et la géographie des plantes cultivées, l'histoire et le mouvement agraire dans les divers pays, l'évolution des techniques agricoles dans le monde ». C'est encore une manifestation du Phénix.

L'élargissement des préoccupations est souligné et ambitieux. La recherche s'universalise ; la pluridisciplinarité apparaît. On va se préoccuper de l'histoire et de la géographie des plantes cultivées et des techniques qui leur sont associées. Une exaltation un peu pompeuse se traduit dans le vocabulaire employé.

«Son ambition est de semer les germes d'une culture internationale de rapprochement entre toutes les nations. [...] C'est aux élites de chaque pays, dans les diverses branches des sciences appliquées et aussi aux travailleurs de la terre qu'il appartient de s'unir et de préparer une rénovation du monde, en se communiquant leurs travaux en vue d'un progrès efficace dont l'humanité a besoin. Des savants désintéressés, des techniciens de l'agriculture, de simples cultivateurs ou planteurs vivant loin les uns des autres [...] peuvent se rendre des services mutuels [...] en faisant connaître leurs techniques, grâce à une revue internationale comme celle-ci. »

Auguste Chevalier a soixante-treize ans, son enthousiasme est intact. On prépare son jubilé : cinquante ans au Muséum ! Il sera célébré le 23 octobre 1947 et un supplément de 59 pages au fascicule 301-302 de 1947 de la RIBA rapportera tous les discours prononcés à cette occasion.

Désormais à la retraite, il publie encore abondamment. En 1951, il signe 51 articles sur les 94 du volume et en 1953, année où il annonce la fin de la revue il a encore 46 articles à son actif sur les 157 au total. Durant plus de trente ans Chevalier n'a cessé de publier, quel que soit le contexte ; même lorsqu'il était en mission pour plusieurs mois. Il envoyait alors des notes de terrain, des lettres et ses collaborateurs restés sur place «distillaient" savamment et très régulièrement des articles écrits avant son départ.

\section{La naissance du JATBA}

\section{La transition - « Mais non! Mais non!»}

L'annonce faite par A. Chevalier dans le bulletin 373-374 de 1953, rapportée dans notre introduction, a pris tout le monde de court.

«Les collègues du Professeur Chevalier ont connu trop tard sa décision pour qu'il leur soit possible d'intervenir utilement car, tous souhaitent très vivement que l'œuvre entreprise depuis 33 années par l'éminent savant ne soit pas abandonnée et continue sous l'impulsion qu'il avait su lui inspirer. » (Vayssière 1954). 

d'agronomie tropicale l'année suivante, travaille sous l'égide de Chevalier au laboratoire en 1928-1929, avant de partir, en 1929, pour l'Afrique où il séjournera pendant près de vingt-huit ans. Il est resté en contact avec le laboratoire, y déposant ses collections de café et de riz notamment. Il a commencé à publier dans la RBA en 1927, avec un article intitulé «La fermentation et la préparation du cacao». On le retrouve avec des articles sur le café, principalement de 1935 à 1940. À partir de la fin de la guerre, c'est le riz qui est son principal sujet de recherche. Ce n'est qu'en 1955, à la fin de la deuxième année de la publication du JATBA, qu'il s'exprime sur le grand changement qui concerne la revue de son laboratoire (Portères 1955). Il parle de Journal d'Agriculture Tropicale, ou de Journal de Botanique appliquée sans jamais citer le nouveau nom exact, comme s'il n'était pas vraiment au courant ou impliqué dans cette affaire. Pourtant le JATBA va devenir son journal et il va lui imprimer sa touche personnelle. Dès ses premières publications, à côté d'articles d'agronomie sur les grandes productions agricoles, il écrit des notes sur des plantes moins importantes économiquement mais qui sont utilisées par les populations locales et sur les techniques agricoles indigènes ${ }^{16}$.

47 La modification progressive du champ d'intérêt couvert par le JATBA correspond à la nouvelle orientation des recherches entreprises au laboratoire. Celle-ci s'est effectuée en grande partie sous l'influence d'André-Georges Haudricourt (1911-1996). 

au laboratoire en 1941, il a travaillé pour la RBA en assurant des traductions d'articles ${ }^{17}$. Il y publie également des articles originaux à partir de cette époque, notamment sur l'origine des plantes cultivées. Ses contributions dans le JATBA sont fréquentes à partir de 1954.

Haudricourt considérait que depuis la fin de la guerre, le développement de plusieurs instituts de recherches consacrés aux productions végétales tropicales (coton, café, cacao, hévéa,...), ne justifiait plus l'existence d'un laboratoire d'Agronomie tropicale au Muséum et il suggéra à Portères de le transformer en laboratoire d'Ethobotanique (ce qui ne deviendra officiel qu'en 1963).

Cependant, dans le tout premier numéro du JATBA, en 1954, apparaît le terme 'ethnobotanique' dans un article de l'agronome Henri Stehlé et plus précisément dans le chapitre VI: «Ethnobotanique précolombienne dans l'archipel caraïbe». En 1954, le terme 'Ethnobotanique' entre également dans l'index par matière publié à la fin de chaque volume : six articles sont répertoriés à cette entrée, onze en 1955. Entre cinq et dix entrées jusqu'en 1960, concernant des résultats de terrain. En 1961 Jacques Rousseau et Roland Portères publient deux articles théoriques sur l'ethnobotanique ${ }^{18}$. À partir de cette date cette discipline sera une des plus traitées dans le JATBA. Ce tournant scientifique que Portères a institutionnalisé en quelque sorte en France et a fait prendre de ce fait à la revue du laboratoire, a été initié, selon lui, par Chevalier. Dans la notice nécrologique qu'il lui consacre, Portères lui rend hommage sur ce point :

«L'originalité de son œuvre c'est de n'avoir pas collecté et étudié les plantes et décrit les végétations seulement pour elles-mêmes, mais toujours dans leurs relations avec l'homme. Il allait encore plus loin et y associait ordinairement les ethnies, les humanités. Il fut, au sens très large et très fidèle du terme, le premier et le plus grand de ceux qui contribuèrent à édifier cette science moderne qu'est l'ethnobotanique » (Portères 1956).

Pourtant Portères en réorientant les recherches au sein du laboratoire va donner une orientation nouvelle à la revue également. : « Si les mots nouveaux n'indiquent guère de changement dans le champ couvert [par la revue], c'est cependant bel et bien d'une conversion scientifique qu'il s'agit, accompagnée de celle qui va s'opérer dans le laboratoire, de la botanique appliquée à l'ethnobotanique. » (Bahuchet \& Lizet $2003: 22$ ). Concernant les sujets traités ces auteurs ajoutent: «...il [Portères] élargit progressivement sa problématique, pour privilégier le plan théorique, puis aborder des aspects très divers des relations des sociétés humaines et du monde végétal, dans une perspective quasi encyclopédique. » (Bahuchet \& Lizet 2003 : 23).

Dans son article de 1955, Portères passe les troupes du laboratoire en revue en citant ses différents collaborateurs et leurs activités scientifiques ou techniques. Il souligne le manque de personnel pour la revue et la bibliothèque. Il finit en annonçant des « orientations nouvelles d'activité » comme notamment la création d'un «Centre de documentation de Botanique et d'Ethno-botanique des Plantes médicinales ». S'il n'est pas totalement impliqué dans la gestion du JATBA, il se préoccupe très tôt de la documentation, tout comme l'a fait Chevalier pendant cinquante ans.

Une nouvelle génération de chercheurs prend en main la revue du laboratoire. Sa gestion est beaucoup plus collégiale que du temps de Chevalier. Un comité de rédaction composé de dix membres est mis en place. Il y a deux rédacteurs en chef, Paul Vayssière et Roland Portères ${ }^{19}$ et deux secrétaires généraux, Jacques Carayon et Jean-François Leroy.

Revue d'ethnoécologie, 1 | 2012 

depuis plusieurs années déjà le laboratoire était baptisé de fait, laboratoire d'Agronomie tropicale. Le nom du laboratoire n'apparaît d'ailleurs sur la couverture du JATBA qu'à partir du changement officiel de nom. Radt (1923-1978).

«Licenciée ès Lettres, documentaliste et bibliographe, excellente traductrice, Charlotte Radt était entrée à la rédaction du JATBA d'abord dans le cadre du personnel du Muséum National d'Histoire Naturelle puis, en 1961, dans le cadre des personnels techniques du CNRS. Elle avait participé activement à la modernisation de notre périodique dont, en outre, elle assurait l'administration. » (Barrau 1977).

De fait, Charlotte Radt a été un des piliers de la revue pendant plus de vingt ans, assurant plus de $90 \%$ des recensions d'ouvrages, en plus de traductions ou d'articles originaux. Pour ce qui concerne la rédaction, une évolution des responsabilités apparait nettement. On a vu que dans les premières années, Portères ne s'est pas beaucoup impliqué et que c'est plutôt Jean-François Leroy qui fait office de rédacteur en chef même si le nom de Paul Vayssière apparaît comme "gérant " sur les bulletins jusqu'à 1970. C'est Leroy qui fait le bilan des dix ans d'existence de la revue en 1964 (Leroy 1964). Portères n'est pas cité dans cet article. « ... dès 1921, A. Chevalier [...] créait la Revue de Botanique Appliquée, celle que nous avons reprise, M. P. Vayssière et moi-même des mains de son fondateur en 1954, sous le titre de Journal d'Agriculture tropicale et de Botanique appliquée. [...] Entrons donc d'un pas assuré dans la deuxième décennie, celle qui nous conduira au cinquantenaire, en 1971, du grand périodique fondé par Auguste Chevalier.»

En 1963 le laboratoire change de nom et devient le laboratoire d'Ethnobotanique. Portères (1965) explique :

«En 1954, le Journal d'Agriculture tropicale et de Botanique appliquée (J.A.T.B.A.) succédant à la R.B.A. évolua progressivement vers ce qui intéressait les connaissances botaniques telles que traditionnalisées dans les Sociétés humaines. Ce périodique est devenu pratiquement une Revue concernant l'Ethnobotanique [... ]. L'orientation prise par le J.A.T.B.A. met en relief l'apport de nouveaux ethnobotanistes de langue française, depuis une dizaine d'années, dont la majorité ne vient plus à cette discipline nouvelle en partant de l'Agronomie, mais de bien d'autres horizons. [...] Comme on peut s'en rendre compte, le titre du J.A.T.B.A. garde toujours sa pleine valeur malgré sa nouvelle orientation, mais à conceptions qui miment (sic.) celles du Laboratoire qui le publie. »

Parmi ces «nouveaux ethnobotanistes", évidemment il faut citer André-Georges Haudricourt (1911-1996).

1965 est aussi l'année de la fondation de la Société d'Ethnozoologie et d'Ethnobotanique $(\mathrm{SEZEB})^{20}$. L'impulsion nouvelle est donnée à la recherche dans le laboratoire et l'ouverture aux chercheurs de multiples disciplines est affirmée. On va constituer des fichiers documentaires et rédiger des fiches méthodologiques propres à cette nouvelle discipline.

«La création récente (1965) d'une Société d'Ethnozoologie et d'Ethnobotanique, exerçant son activité au Laboratoire d'Ethnobotanique, est encore une indication de l'intérêt porté à ce genre d'études. Fruit d'une coopération entre Naturalistes, Ethnologues et Linguistes, il est probable qu'elle rendra de grands services à différentes disciplines de recherche, et en définitive à la connaissance de ce que furent, sont et seront les relations entre les êtres vivants et les Sociétés humaines. 
Ainsi, la conversion progressive du laboratoire d'Ethnobotanique commencée en

1962, fournit une unité de travail d'un aspect nouveau en Europe ». (Portères 1965). en main et signe un éditorial intitulé : « Visage nouveau... Vie nouvelle !» (Barrau 1973). En réalité la revue a besoin d'un nouveau souffle et les retards de publication s'accumulent depuis plusieurs années. On change de look. Barrau s'en réfère au passé, aux ancêtres « Nous sommes certains de poursuivre [...] l'œuvre de nos prédécesseurs » tout en exaltant une « ethnobotanique française qui se porte bien et va de conserve avec cette ethnozoologie sur laquelle A.-G. Haudricourt avait attiré l'attention de la recherche française dans un article que L'Homme publia en $1962^{21}$ ». La rédaction ajoute : «À titre exceptionnel et pour rattraper le retard pris par notre Revue, nous publions ce fascicule unique pour 1973. Le rythme et la forme normale de notre Publication reprendront très prochainement avec le no 1-2-3, Janvier-Février-Mars 1974 ». C'est l'année où Roland Portères va mourir. Jacques Barrau annonce ce décès dans le fascicule 4-5-6 de 1974 et publie en 1976 : Travaux de Roland Portères publiés dans le JATBA depuis 1954 classés par ordre chronologique.

\section{Rien ne va plus}

61 Le retard ne sera pas complètement épongé et se reportera pendant plusieurs années encore ; le deuxième et dernier fascicule de 1979 porte la mention de dépôt légal au $2^{\text {ème }}$ trimestre 1980.

Entre-temps la rédaction a subi des changements importants. En 1977, il y a trois directeurs: Lucien Bernot, André-Georges Haudricourt et Jean-François Leroy, un rédacteur en chef, Jacques Barrau, une rédactrice et une secrétaire de rédaction. Un Conseil de rédaction de neuf membres dont deux américains est formé. Jacques Barrau signe un éditorial dans lequel il souligne les difficultés techniques et surtout financières de la revue. Il parle de «combat pour la survie » et en 1978, de « retard considérable » dans la parution.

En 1979, un ingénieur d'études du CNRS est affecté à la rédaction de la revue. Un changement d'imprimeur a lieu. Un nouveau texte d'accueil apparaît en deuxième de couverture qui parle de "carrefour des sciences de la nature et des sciences de l'homme ». Un nouveau changement d'imprimeur a lieu en 1981 traduisant sans doute les difficultés de trouver des fonds. En 1983, l'administration scientifique et technique de la revue se complexifie encore. La revue est publiée avec l'aide du CNRS et de la Fondation de la Maison des Sciences de l'Homme ${ }^{22}$.

64

En 1984 il est fait appel à l'ORSTOM pour financer la revue. En contrepartie un chercheur de cette institution entre au Comité de Direction (D. Spire) et trois autres au Comité de Rédaction. Cette collaboration dure deux années. 

et est intitulé Un terrien des ̂̂les : à propos de Jacques Barrau. Il est dirigé par Alice Peeters. C'est un numéro phénix aussi. La nouvelle direction du Muséum fait savoir que cette parution est juridiquement et financièrement dans l'illégalité et qu'il n'est plus question de faire sortir un numéro qui ne soit pas édité par les Publications scientifiques du Muséum. Des discussions sont engagées... Et le phénix est là !

Les noms de la RBA

Les noms de la $R B A$

\section{BIBLIOGRAPHIE}

Bahuchet S. \& Lizet B. 2003 - L'ethnobotanique au Muséum national d'histoire naturelle. Les hommes, les idées, les structures. In Lieutaghi P. \& Musset D. (Dir.), Plantes, sociétés, savoirs, symboles. Matériaux pour une ethnobotanique européenne, Actes du séminaire d'Ethnobotanique de Salagon. Premier volume, année 2001. Mane, les Alpes de lumière : 15-32. (Les cahiers de Salagon 8). Baillaud E. 1907 - Jean Vilbouchevitch. Journal d'Agriculture Tropicale 68 : 36-39.

Barrau J. 1970 - Activités du laboratoire d'ethnobotanique. Une exposition ethnobotanique en Haute-Provence. Journal d'Agriculture Tropicale et de Botanique Appliquée 17 (7-8-9) : 342-345. 
Barrau J. 197724 - Charlotte Radt (1923-1978). Journal d'Agriculture Tropicale et de Botanique Appliquée 24 (4) : 284.

Barrau J. 1987 - Cher abonné, cher lecteur.... Journal d'Agriculture Traditionnelle et de Botanique Appliquée $34: 5$.

Bonneuil C. 1991 - Des savants pour l'empire. La structuration des recherches scientifiques coloniales au temps de "la mise en valeur des colonies françaises", 1917-1945. Bondy, Éditions de l'ORSTOM, 123 p. (Collection Études et thèses).

Bonneuil C. 1996 - Auguste Chevalier, savant colonial : entre science et Empire, entre botanique et agronomie. In Petitjean P. (Ed.), Les sciences hors d'occident au $x x^{e}$ siècle, vol. 2 : Les sciences coloniales figures et institutions. Paris, ORSTOM Éditions : 16-35.

Bobichon H. 1926-1927 - Procès-Verbaux des séances du comité de patronage du laboratoire d'agronomie coloniale, manuscrit, p. 111.

Bulletin de la Société d'Ethnozoologie et d'Ethnobotanique 1968 - La société d'Ethnozoologie et d'Ethnobotanique 1 : 1-3.

Chevalier A. 1921 - Fonctionnement du Laboratoire d'Agronomie coloniale pendant la guerre et jusqu'au 31 décembre. Revue de Botanique appliquée et d'Agriculture coloniale 1 (1) : 3-11.

Chevalier A. 1924 - Notre programme pour 1924. Revue de Botanique appliquée et d'Agriculture coloniale 4 (29) : 1-3.

Chevalier A. 1925 - Règles à observer par les Collaborateurs de la Revue. Revue de Botanique appliquée et d'Agriculture coloniale 5 (41) : 1-14.

Chevalier A. 1929 - La Revue de Botanique appliquée entre dans sa neuvième année. Revue de Botanique appliquée et d'Agriculture tropicale 9 (89) : 1-2.

Chevalier A. 1943 - Historique de la Revue de Botanique appliquée. Volonté d'en continuer la publication. Revue de Botanique appliquée et d'Agriculture tropicale 23 (257-258-259) : 1-6.

Chevalier A. 1945 - La Revue de Botanique appliquée et d'Agriculture tropicale et son programme. Revue de Botanique appliquée et d'Agriculture tropicale 25 (275-276) : 1-3.

Chevalier A. 1946 - Revue Internationale de Botanique appliquée et d'Agriculture tropicale. Programme. Revue internationale de Botanique appliquée et d'Agriculture tropicale 26 (279-280) : 1-2.

Chevalier A. 1947 - Réponse de M. Chevalier. In Jubilé de M. Auguste Chevalier célébré à Paris au Muséum national d'Histoire naturelle le 23 octobre 1947 : compte-rendu, discours, adresses. Revue internationale de Botanique appliquée et d'Agriculture tropicale, Supplément au nº 301-302 (novembre-décembre 1947) : 39-49.

Chevalier A. 1953 - Fin de la publication de la Revue (Courte notice sur l'historique de la Revue Internationale de Botanique Appliquée et d'Agriculture tropicale). Revue Internationale de Botanique Appliquée et d'Agriculture tropicale : 373-374 : 477-478.

Compte-rendu sommaire de l'Association des Botanistes du Muséum $\mathrm{n}^{\circ} 1$ - Revue de Botanique Appliquée et d'Agriculture tropicale, 1933, 13 (137) : 93.

Guédès M. 1974 - Roland Portères (1906-1974). Histoire et nature 2 : 3-4.

Kleiche M. 1995 - La professionnalisation des agronomes coloniaux français : L'École de Nogent, 1902-1940. In Chatelin Y. \& Bonneuil C. (Ed.), Les sciences hors d'occident au xx ${ }^{e}$ siècle, vol. 3, Nature et environnement. Paris, ORSTOM Éditions : 75-89. 
Leroy J.-F. 1954 - La vie et l'œuvre du Professeur Auguste Chevalier. Journal d'Agriculture Tropicale et de Botanique appliquée $1: 3-7$.

Leroy J.-F. 1964 - Le Journal d'Agriculture Tropicale et de Botanique Appliquée ou Dix ans de vie nouvelle (1954-1964). Journal d'Agriculture Tropicale et de Botanique appliquée 11 (1-3) : 1-2.

Note de la rédaction 1925 - Fondation de l'Association. Actes et Comptes-rendus de l'Association Colonies-Sciences 1 (1) : 1 .

Portères R. 1955 - Activité du laboratoire d'Agronomie tropicale en 1955. Journal d'Agriculture Tropicale et de Botanique appliquée 2 : 687-690.

Portères R. 1956 - Auguste Chevalier (1873-1956), Journal d'Agriculture Tropicale et de Botanique appliquée 3 (3-4) : 218-219.

Portères R. 1961 - L'ethnobotanique : place - objet - méthode - philosophie. Journal d'Agriculture Tropicale et de Botanique appliquée 8 (4-5) : 102-109.

Portères R. 1965 - Le Laboratoire d'Ethnobotanique du Muséum national d'Histoire naturelle (Paris). Journal d'Agriculture Tropicale et de Botanique appliquée 12 (1-2) : 1-4.

Rousseau J. 1961 - Le champ de l'ethnobotanique. Journal d'Agriculture Tropicale et de Botanique appliquée 8 (4-5) : 93-101.

Stehlé H. 1954 - Quelques notes sur la botanique et l'écologie végétale de l'Archipel des Caraïbes. Journal d'Agriculture Tropicale et de Botanique appliquée 1 : 71-110.

Urbain A. 1947 - Discours de M. le Professeur Ach. Urbain : la carrière du Professeur Chevalier. In Jubilé de M. Auguste Chevalier célébré à Paris au Muséum national d'Histoire naturelle le 23 octobre 1947 : compte-rendu, discours, adresses. Revue internationale de Botanique appliquée et d'Agriculture tropicale, Supplément au nº 301-302 (novembre-décembre 1947) : 13-21.

Vayssière P. 1954 - Avant-propos. Journal d'Agriculture tropicale et de Botanique appliquée $1: 1$.

Vogüé A. de 1928-1941 - Procès-Verbaux des séances du Comité de patronage du laboratoire d'agronomie coloniale. Manuscrit, p. [141].

\section{ANNEXES}

\section{Quelques articles remarquables}

1928 Annonce de la création de l'Institut International d'Agriculture de Rome, ancêtre de la FAO.

1930 Plusieurs articles sur la photo aérienne dont ce sont les vrais grands débuts. Marcel Griaule publie un article. "La culture indigène du Caféier dans les provinces du nord de l'Abyssinie ». Chevalier fait le vœu relatif à la Protection des types sauvages de plantes cultivées ou des types peu améliorés cultivés par les indigènes.

1931 Un article de Paul Rivet dans les CR de l'Association Colonies-Sciences

1932 Critique acerbe de l'exposition coloniale de Vincennes 1931. Article de Louis Hédin : « La vocation des terres de la forêt tropicale d'après les indices fournis par la végétation spontanée ; observations des indigènes sur la flore et les formations végétales ». Premier article sur l'origine des riz.

1934 Préparation des collections botaniques et forestières dans les pays tropicaux

1937 Décès de Madame Philippe de Vilmorin née Mélanie de Dortan. La constitution du 
Musée de l'Homme est signalée dans les CR de l'Association Colonies-Sciences 1940 Trois articles de A.-G. Haudricourt. L'agriculture indigène est l'objet de onze articles

1943 Établissement d'un catalogue de la bibliothèque. Établissement d'une « Liste alphabétique des genres espèces et variétés des plantes nouvelles ou peu connues décrites dans la Revue »

1947 Récit de la guerre au Jardin des plantes par Auguste Chevalier. Jubilé scientifique de Auguste Chevalier

1948 Annonce de la création de la Revue de Géographie humaine et d'Ethnologie, ISSN

1141-2119.

1956 Premier article de Jacques Barrau : « Plantes alimentaires de base des mélanésiens », JATBA 3 (1-2) : 32-49.

\section{Numéros spéciaux de la $R B A$}

Ils sont tous signés Auguste Chevalier sauf celui concernant l'Amiral Dumont d'Urville qu'il a dirigé.

1928 Monographie de l'Arachide. Première partie : l'Arachide en général, 13 (146-147) : 689-789.

1929 Monographie de l'Arachide. Première partie : l'Arachide en général, 14 (156) : 565-632 ;

(157) : 709-755 ; (158) : 833-864.

1929 L'Agronomie coloniale et le Muséum d'Histoire naturelle/Conférences du cours de Auguste Chevalier au Muséum en 1930, 10 (106bis) : 425-552.

1932 Les Productions végétales du Sahara et de ses confins Nord et Sud : passé - présent - avenir, 12 (133-134) : 669-924.

1935 Les Îles du Cap Vert : géographie, biogéographie, agriculture. Flore de l'Archipel 15

(170-171) : 733-1090.

1936 Monographie de l'Arachide. Deuxième partie : l'Arachide au Sénégal 16 (181-182) :

673-872. Plus de 760 références bibliographiques.

1946 Cinquantenaire de Madagascar. Cinquante années d'efforts scientifiques et sociaux pour le développement de l'agriculture malgache 26 (286bis) : 333-504.

1951 Au souvenir de l'Amiral J. Dumont d'Urville, prospecteur de la flore de l'Océanie et de l'Hémisphère austral de 1822 à 184031 (339-340) : 136 p.

\section{Numéros spéciaux du JATBA}

Adam J.G. : Noms vernaculaires des plantes du Sénégal, 112 p.

Adam J.G. : Itinéraires botaniques en Afrique occidentale, 200 p.

Adam J.G., Échard N. \& Lescot M. : Plantes médicinales Hausa de l'Ader (République du Niger), $141 \mathrm{p}$

Bosseaux M. E. : Végétation et flore des îles Saint Pierre et Miquelon, 103 p.

Botton H. : Les plantes de couverture en Côte d'Ivoire, 194 p.

Bruneau M. \& Bernot L. : Une population lacustre : les Intha du Lac Inlé (États Shan du sud, Birmanie), $40 \mathrm{p}$

Bruneau de Miré P. \& Gillet H. : Contribution à l'étude de la flore du Massif de l'Aïr, 660 p. Chevalier A. : Monographie de l'arachide : l'arachide au Sénégal, 200 p.

Chevalier A. : Cinquantenaire de Madagascar : cinquante années d'efforts scientifiques et sociaux pour le développement de l'Agriculture Malgache, $170 \mathrm{p}$.

Dournes J. : Chi-Chê la botanique des Srê, 190 p. 
Dournes J. : Bois-bambou (Köyau-ale) : aspect végétal de l'univers jörai, 196 p.

Dubois M.J. : Ethnobotanique des Maré, Îles Loyauté (Nouvelle Calédonie), 116 p.

Ferrari J.-P. \& Aillaud G. : Bibliographie du genre Capsicum

Gillet H. : Étude des pâturages du Ranch de l'Ouadi-Rimé. Tchad, 158 p.

Gillet H. : Pâturages sahéliens. Le Ranch de l'Ouadi-Rimé, 210 p.

Girard F. : Les gens de l'igname : les Buang de la vallée du Snake, District de Morobé, Nouvelle-Guinée, $50 \mathrm{p}$.

Jacques-Félix H. : Contribution de René Caillé à l'ethnobotanique africaine au cours de ses voyages en Mauritanie et à Tombouctou 1819-1828, $172 \mathrm{p}$.

Kerharo J. \& Adam J.G. : Plantes médicinales et toxiques des Peul et des Toucouleur du Sénégal, $160 \mathrm{p}$.

Kupzow A.J. : Histoire du maïs, 35 p.

Lavauden L. : Les forêts coloniales de la France, 280 p.

Lemordant D. : Contribution à l'ethnobotanique éthiopienne, $140 \mathrm{p}$.

Lyman Thomas Amis: Miao (Meo) slash-and-burn agriculture, $35 \mathrm{p}$.

Matras J. \& Martin M.A. : Contribution à l'ethnobotanique des Brou (Cambodge - Province de RatanaKiri), $100 \mathrm{p}$.

Métailié G. (dir.) :Agriculture chinoise traditionnelle, $280 \mathrm{p}$.

Panoff M. (dir.) : Tubercules et pouvoir, $150 \mathrm{p}$.

Poilane E. : Les arbres fruitiers d'Indochine, $100 \mathrm{p}$.

Portères R. : Les céréales mineures du genre Digitaria en Afrique et en Europe, 100 p.

Portères R. : Compagnonnage agraire et riz cultivés

Portères R. : Le nom des riz en Guinée, 346 p.

Portères R. : Les appellations des céréales en Afrique, 286 p.

Portères R. : Taxonomie agrobotanique des riz cultivés 0 . sativa Linné et 0 . glaberrima

Steudel, $260 \mathrm{p}$.

Sigaut F. : Les hommes et leurs sols : les techniques de préparation du champ dans le fonctionnement et dans l'histoire des systèmes de culture, $220 \mathrm{p}$.

Vergiat A.M. : Plantes magiques et médicinales des féticheurs de l'Oubangui (Région de Bangui), $238 \mathrm{p}$.

Wong M. : Contribution à l'histoire de la matière médicale végétale chinoise, $301 \mathrm{p}$.

\section{Quelques chiffres}

Nombre de pages de la Revue de Botanique appliquée (RBA) et des Comptes rendus de l'Association Colonies-Sciences (ACS)

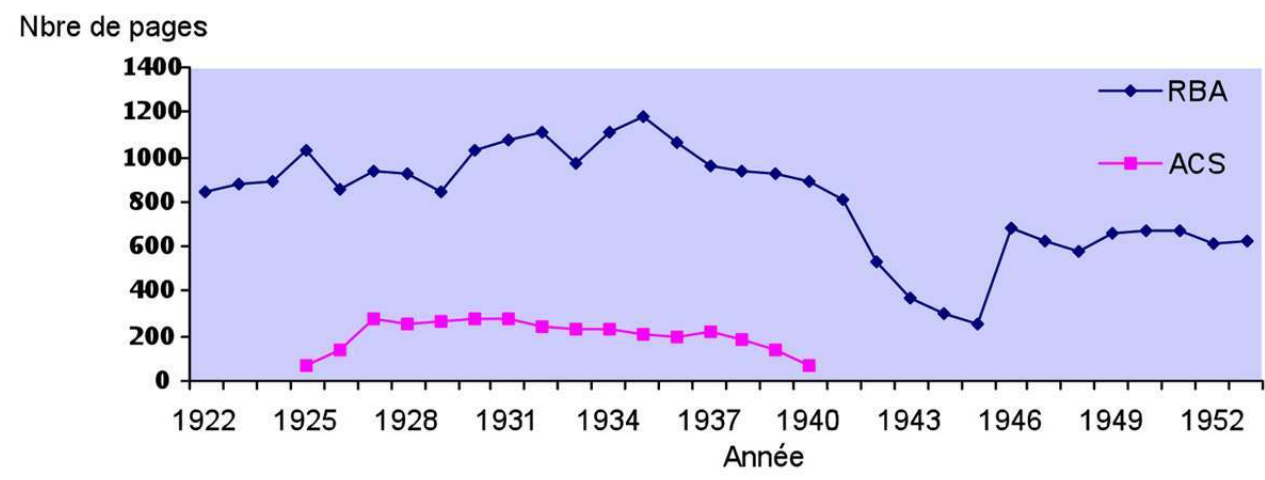


Nombre de pages du Journal d'Agriculture Traditionnelle et de Botanique Appliquée

Nbre de pages
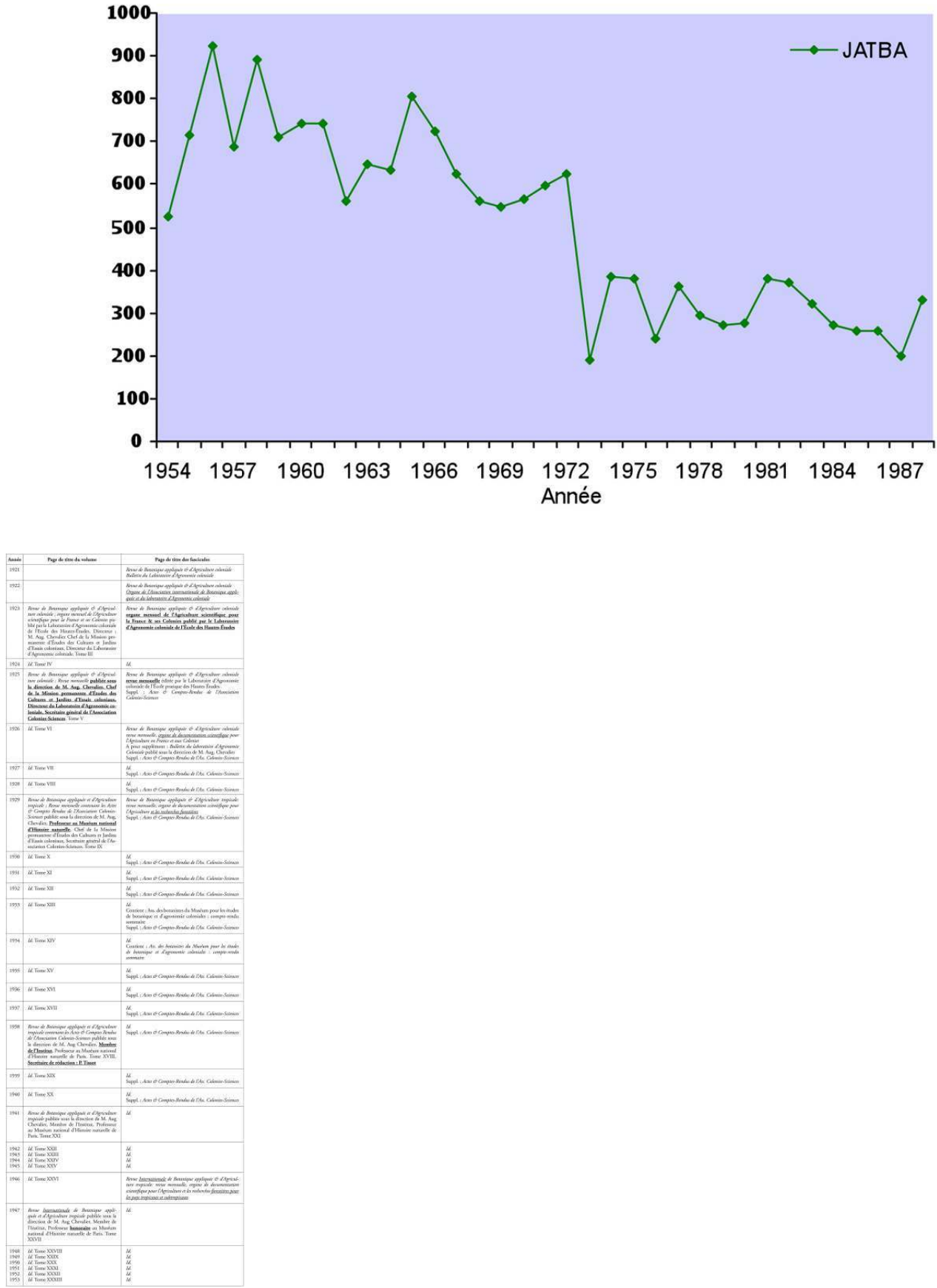

En souligné : les changements intervenus

Les noms du JATBA 


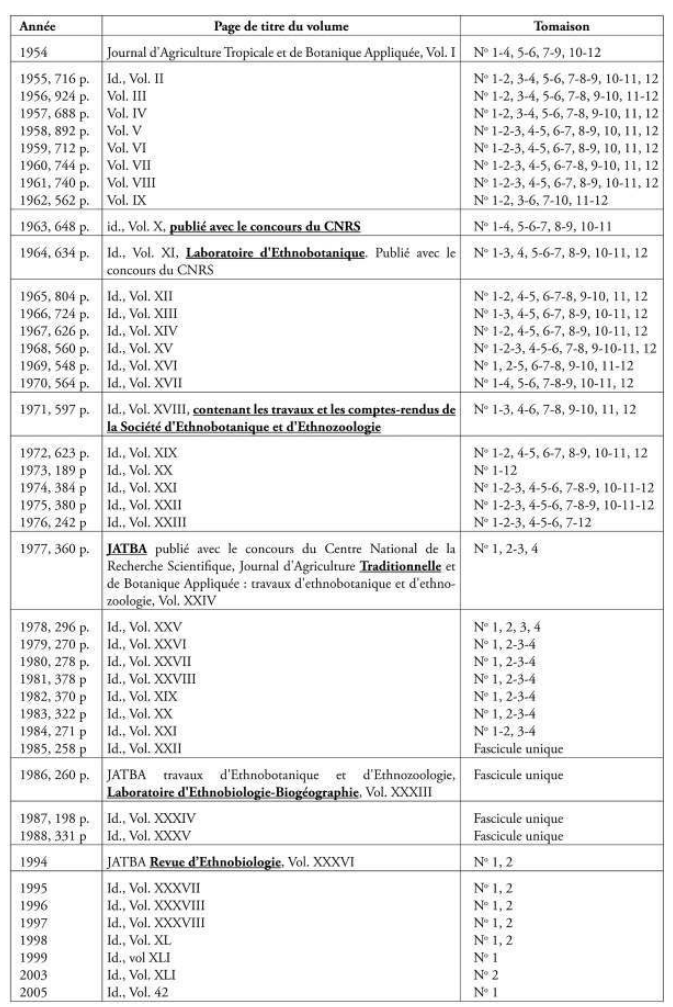

En souligné : les changements intervenus

\section{Publications satellites du JATBA}

Une collection apparaît en 1972 :

Cahier du journal d'agriculture tropicale et de botanique appliquée ISSN 0764-9037

No 1 Allain Patrick, 1972 : Phénomènes culturels et mondes de l'imaginaire : cannabis et peyotl, $143 \mathrm{p}$.

No 2 Vidal Jules, 1972 : Contribution à l'ethnobotanique du Laos et du Cambodge (noms vernaculaires, usages), $88 \mathrm{p}$.

No 3 Luxereau Anne, 1972 : Étude ethnozoologique du Pays Hausa en République du Niger, $341 \mathrm{p}$.

No 5. Antaki Ikram, 1973 : Deir-Atieh (Syrie). Essai d'ethnographie rurale, 146 p.

Pas de trace du No 4

Le 5 est devenu le 4 après quelques années

Un périodique paraît sous plusieurs titres pendant quelques années :

Histoire et biologie 1968-1969 ISSN 0441-6732

Histoire et Nature 1973-1993 ISSN 0396-9681

Plusieurs bulletins associés à la création aux activités de la SEZEB :

Bulletin de la Société d'Ethnozoologie et d'Ethnobotanique, 1968, 1

Bulletin de liaison de la Société d'Ethnozoologie et d'Ethnobotanique, 1976-1987

Supplément au Bulletin d'information de la Société d'ethnozoologie et d'ethnobotanique, 1969-1977, ISSN 09901663

Bulletin bibliographique de la Société d'ethnozoologie et d'ethnobotanique, 1978-1980, ISSN 09901701. 


\section{Constitution de la bibliothèque}

En 1943, $23^{\text {ème }}$ année de la publication de la revue, Auguste Chevalier écrit un article intitulé « Historique de la Revue de Botanique Appliquée et d'Agriculture Tropicale : volonté d'en continuer la publication ", dans lequel il parle de la constitution de la bibliothèque. Évoquant la cessation de parution de la Revue des cultures coloniales dirigée par Milhe-Poutingon : «[...] en 1904, [...] Milhe-Poutingon souffrant, [...] nous fit don [...] des publications sur les cultures tropicales rassemblées par lui ; elles ont constitué le premier noyau d'une bibliothèque d'agronomie coloniale que nous avons fondée au Muséum. » Dans le même article un peu plus loin, évoquant la disparition de Vilbouchevitch et de son Journal d'Agriculture tropicale, il signale :

« Mme Nageotte-Vilbouchevitch voulut bien donner à mon laboratoire ce qui restait de l'importante bibliothèque rassemblée par son frère et de précieuses publications vinrent ainsi rejoindre celles qui avaient été réunies par la Revue des cultures coloniales. Telle est l'origine du fonds de début de la Bibliothèque d'Agriculture coloniale que j'ai constituée au Laboratoire d'Agronomie coloniale du Muséum. »

Plus loin, parlant du rayonnement de sa revue :

«En 1940, elle était lue dans presque toutes les régions du globe. Nous l'échangions avec les publications des Stations expérimentales existant dans la plupart des pays tropicaux et subtropicaux, ce qui a permis d'étendre considérablement la bibliothèque du Laboratoire. »

\section{NOTES}

1. Il n'existait jamais qu'un seul phénix à la fois : il vivait très longtemps ; quand il sentait sa fin venir, [il] construisait un nid de branches aromatiques et d'encens, y mettait le feu et se consumait dans les flammes. Des cendres de ce bûcher surgissait un nouveau phénix. (Larousse [1901]).

2. L'Union coloniale française (UCF), fut fondée en 1893 dans l'esprit d'une chambre du commerce colonial rassemblant l'ensemble des intérêts économiques coloniaux. Très riche, sous l'impulsion de son directeur général Joseph Chailley, elle utilisa ses immenses moyens à l'organisation d'une structure administrative stable et compétente dotée de services pour chaque domaine, jouant un rôle d'office colonial (Bonneuil 1991).

3. « ... je fus embrigadé contre mon gré dans une étrange aventure » (Chevalier 1947).

4. Jean Vilbouchevitch (1866-1907), agronome russe installé en France depuis 1895, a d'abord été attaché à la Revue des Cultures coloniales avant de créer son propre titre (Baillaud 1907).

5. Ce sont les numéros de récolte et chacun peut donner lieu à plusieurs " parts ", c'est-à-dire à plusieurs planches d'herbier. Cette association est l'ancienne Académie internationale de Géographie botanique fondée en 1890 par $\mathrm{M}^{\mathrm{gr}}$ Léveillé et dont A. Chevalier est devenu le président en 1919. Il semble que Chevalier veuille faire apparaître les multiples responsabilités qui sont les siennes dans ces sous-titres qui changent et changeront encore bien souvent.

6. Pour la chronologie des parutions et les changements de titres de la revue, voir annexe.

7. À la différence de la Revue d'Agronomie Coloniale animée par l'Institut national d'agronomie coloniale de Nogent-sur-Marne, celle-ci [la RBA] donne de larges comptes rendus des recherches menées dans les possessions et les pays tropicaux étrangers. (Bonneuil 1996)

8. Cette différence tient sans doute au fait que le bulletin no 1 est considéré comme étant paru, tantôt en 1913 en suppl. au Jd'AT, tantôt en 1921, dans le volume I de la RBA. 
9. Cette association est l'ancienne Académie internationale de Géographie botanique fondée en 1890 par Mgr Léveillé et dont A. Chevalier est devenu le président en 1919. Il semble que Chevalier veuille faire apparaitre les multiples responsabilités qui sont les siennes dans ces soustitres qui changent et changeront encore bien souvent.

10. C'est la première apparition du sigle RBA qui, on le remarque, ne recouvre pas entièrement le titre de la revue.

11. L'année de l'indépendance de la Guinée, il était temps!

12. En 1927 il a passé deux mois en Afrique du Nord, en 1928, cinq mois au Brésil, puis ce sont plusieurs missions en Afrique de l'Ouest entre 1929 et 1932 et aussi les Îles du Cap Vert et les Canaries pendant cinq mois en 1934.

13. Journal des Opérations Diverses du Comité de Patronage du Laboratoire d'Agronomie coloniale du mois de juin 1933 au mois de Décembre $1943:$ 20-95.

14. 1922 : « Notre programme»; 1924 : « Notre programme pour $1924 » ; 1927$ : «Aux lecteurs de la Revue de Botanique appliquée et aux Amis du Laboratoire d'Agronomie coloniale »; 1929 : «La RBA entre dans sa neuvième année »; 1930: «La dixième année de la RBA »; 1941: "La RBA entre dans sa IIIème décade »; 1943: "Historique de la Revue de Botanique appliquée et d'Agriculture traditionnelle: volonté d'en continuer la publication»; 1945: «La Revue de Botanique appliquée et d'Agriculture traditionnelle et son programme»; 1946: «Revue Internationale de Botanique appliquée et d'Agriculture traditionnelle : Programme ».

15. Cf. le «Journal des Opérations du Comité de Patronage du laboratoire d'Agronomie coloniale », octobre 1941 à juin 1944 », p. 198 à 262.

16. Plantes toxiques utilisées par les peuplades Dan et Guéré de la Côte d'Ivoire, Bulletin du Comité d'Études Historiques et Scientifiques de l'Afrique Occidentale Française, 1936, 18 (1) : 13 p. Notes sur la riziculture indigène du Nord-Ouest de la Côte d'ivoire, Bulletin du Comité d'Études Historiques et Scientifiques de l'Afrique Occidentale Française, 1936, 18 (1) : 92-127.

17. Il émarge au Comité de Patronage de mars 1941 jusqu'à décembre 1942 pour la somme de 200 francs sous la rubrique Personnel, sous-rubrique Gratifications. Journal des Opérations du Comité de Patronage du laboratoire d'Agronomie coloniale, mars 1941 à décembre 1942 : p. 184 à 225.

18. Voir bibliographie.

19. Marquant ainsi les deux orientations principales qu'est censée prendre la revue: l'entomologie et l'agronomie appliquées aux régions tropicales.

20. Selon les statuts, cette société est constituée «à l'effet de procéder d'abord à la constitution d'un fichier interdisciplinaire, ethnologique, linguistique, ethnobotanique et ethnozoologique, d'effectuer la recherche et la collecte, par des missions scientifiques, des matériaux et de la documentation nécessaire, de fournir à tous chercheurs le maximum d'informations correspondant à leurs travaux et orientation en matière d'Ethnobotanique et d'Ethnozoologie ». (Bulletin de la SEZEB 1968). Ses membres fondateurs sont Jacqueline M.C. Thomas, linguiste, Roland Portères et André-Georges Haudricourt.

21. A.-G. Haudricourt, " Domestication des animaux, culture des plantes et traitement d'autrui », L'Homme 2(1), 1962. Sans doute est-ce pour cela que Portères situe la conversion progressive à partir de 1962.

22. Il y a : un Conseil de Direction : Bernot, Haudricourt et Leroy avec comme rédactrice en chef Chantal Gaulin, comme secrétaire générale à la rédaction Claudine Friedberg et comme assistante à la rédaction Françoise Aubaile-Sallenave; un Conseil de Rédaction de neuf membres et un Comité de Rédaction composé de sept chercheurs du Muséum.

23. 1994 : Diversité culturelle; diversité biologique, textes réunis par Jacques Barrau et Bernadette Lizet ; 1996 : Biodiversité, friches et jachères, textes réunis par Paul Arnould, Bernard Roussel et Claude Sastre; Ethnozoologie en hommage au Professeur Pujol; 1997 : Sauvages dans la ville: de l'inventaire naturaliste à l'écologie urbaine, hommage à Paul Jovet, textes réunis par Bernadette Lizet, Anne-Elizabeth Wolf et John Celecia ; 1998 : Conserver, gérer la biodiversité quelle stratégie pour la 
Guyane? textes réunis par Marie Fleury et Odile Poncy; 1999 : Systèmes de culture traditionnels et pratiques paysannes nouvelles en Océanie, textes réunis par Annie Walter et Alain Leplaideur.

24. On remarque le décalage de la date de publication avec la date du décès de $C$. Radt, dû au retard de publication de la revue.

\section{RÉSUMÉS}

Le botaniste Auguste Chevalier, explorateur de l'Afrique au début du xx siècle, crée en 1921 la Revue de Botanique appliquée et d'Agriculture coloniale. Au lendemain de la Première Guerre mondiale, les pays européens se lancent dans des politiques de «mise en valeur » des colonies et cherchent de nouveaux produits rentables. La revue s'intéressera à l'agriculture, l'horticulture, la foresterie. Pendant plus de trente ans Chevalier dirige le laboratoire d'Agronomie coloniale du Muséum national d'Histoire naturelle ainsi que cette publication scientifique, rédigeant souvent lui-même une grande partie de son contenu.

En 1954, la revue devient le Journal d'Agriculture tropicale et de Botanique appliquée. Autres titres, autres directeurs, autres contenus. La revue accompagne alors la naissance de l'ethnobotanique française. Mais aux périodes fastes des années 1930 ont succédé des années plus difficiles, parfois des éclipses, des aléas financiers, politiques, scientifiques émaillant l'histoire de ce périodique. Le JATBA tente aujourd'hui un nouveau départ et c'est son histoire que nous retraçons ici.

Botanist Auguste Chevalier, explorer in Africa in the early $20^{\text {th }}$ century, founded the Revue de Botanique appliquée et d'Agriculture coloniale in 1921. Just after WW1, European countries launched policies to "call attention" to the colonies and sought new cost-effective products. The journal dealt with agriculture, horticulture and forestry issues. For over 30 years, Chevalier was at the head of the Colonial Agronomy laboratory of the Muséum National d'Histoire Naturelle, and supervised this scientific publication, often writing a large part of its contents himself.

In 1954, the journal became the Journal d'Agriculture tropicale et de Botanique Appliquée. New headings, new editors, new contents. The journal then supported the emergence of French ethnobotany. Yet, harder years followed the prosperous period of the 30's, with occasional lacks, financial, political and scientific hazards distinguishing the history of this periodical. JATBA is now attempting a fresh start and we shall try to recount its history in this paper.

\section{INDEX}

Mots-clés : revue, agriculture, agronomie coloniale, botanique, ethnobotanique, histoire, Chevalier (Auguste)

Keywords : journal, agriculture, colonial agronomy, botany, ethnobotany, history, Chevalier (Auguste) 


\section{AUTEUR}

\section{CATHERINE HOARE}

UMR 7206, Éco-Anthropologie et Ethnobiologie

Muséum national d'histoire naturelle

Département Hommes, Natures, Sociétés

case postale 26, 57 rue Cuvier, 75005 Paris (France)

hoare@mnhn.fr 